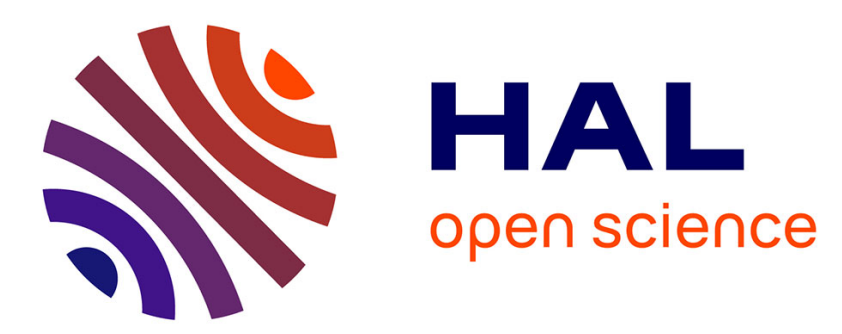

\title{
Mobilité, transports et territoires dans la science régionale francophone : illustrations par quelques articles. Deuxième partie.
}

Guy Joignaux, Lourdes Diaz Olvera

\section{- To cite this version:}

Guy Joignaux, Lourdes Diaz Olvera. Mobilité, transports et territoires dans la science régionale francophone : illustrations par quelques articles. Deuxième partie.. RTS - Recherche Transports Sécurité, 2013, Mobilité, transports et territoires, 2013 (01), pp.61-62. 10.4074/S0761898013001052 . hal-01670623

\section{HAL Id: hal-01670623 \\ https://hal.science/hal-01670623}

Submitted on 21 Dec 2017

HAL is a multi-disciplinary open access archive for the deposit and dissemination of scientific research documents, whether they are published or not. The documents may come from teaching and research institutions in France or abroad, or from public or private research centers.
L'archive ouverte pluridisciplinaire HAL, est destinée au dépôt et à la diffusion de documents scientifiques de niveau recherche, publiés ou non, émanant des établissements d'enseignement et de recherche français ou étrangers, des laboratoires publics ou privés. 


\title{
Mobilité, transports et territoires dans la science régionale francophone : illustrations par quelques articles. Deuxième partie.
}

\author{
Mobility, transport and territories in the French-speaking regional science community : depicted \\ in some articles. Part two
}

\author{
Guy Joignaux · Lourdes Diaz Olvera \\ (C) IFSTTAR et Éditions NecPlus 2013
}

Une première livraison de quatre articles issus de présentations faites au $48^{\mathrm{e}}$ Colloque de l'Association de science régionale de langue française (ASRDLF) (juillet 2011 à Schoelcher, en Martinique) a fait l'objet d'une publication dans le précédent RTS. Cette livraison était précédée d'une description de l'ensemble des travaux développés dans le cadre de ce colloque dans les sessions consacrées aux thématiques Transport, mobilité et territoires, illustrant la richesse et l'importance de ces sujets au sein de cette communauté scientifique. Elle est à présent complétée par deux autres articles, brièvement replacés dans leur contexte ci-après.

Dans le premier article, Stéphanie Souche et Aurélie Mercier abordent la simulation de l'effet d'un péage de cordon sur l'évolution à court terme des inégalités sociales et spatiales dans l'agglomération de Lyon. La simulation porte sur la comparaison de la situation avant-après la mise en place du péage de cordon de 5 euros autour de la ville-centre de l'agglomération (Lyon-Villeurbanne), où sont concentrés $60 \%$ des emplois de l'agglomération. Les auteures entreprennent cet exercice à travers une démarche en trois volets : la mesure des inégalités sociales selon trois indicateurs traditionnels (Gini, Theil et Atkinson); la mesure de l'accessibilité potentielle aux emplois, en y

Articles issus du $48^{\mathrm{e}}$ Colloque de l'Association de science régionale de langue française, Schoelcher (Martinique), juillet 2011, sessions Transport, Mobilités et Territoires.

Guy Joignaux $(\bowtie)$

Univ Lille Nord de France, F-59000, Lille, France IFSTTAR

BP 70317, F-59666 Villeneuve d'Ascq Cedex

e-mail : guy.joignaux@ifsttar.fr

Lourdes Diaz Olevra $(\triangle)$

Université de Lyon, F-69000, Lyon, France

CNRS, LET, F-69007, Lyon

ENTPE, LET, F-69518, Vaulx-en-Velin

14 avenue Berthelot, F-69007 Lyon

e-mail : lourdes.diaz-olvera@entpe.fr introduisant également le coût temporel et le coût monétaire des déplacements domicile-travail ; et enfin, la prise en compte des impacts du péage selon deux échelles spatiales, le découpage de l'agglomération en zones et l'ensemble de l'agglomération. La simulation s'appuie sur les données issues de l'enquête-ménages déplacements de 2006, en se limitant pour ces analyses au périmètre défini par le Schéma de cohérence territoriale lyonnais.

Les résultats montrent que l'évolution des inégalités de revenu varie sensiblement selon l'indicateur utilisé, avec des nuances selon l'échelle étudiée. Selon l'indice de Gini, les inégalités augmentent, quelle que soit l'échelle spatiale. De manière similaire, l'indicateur d'Atkinson montre l'augmentation des inégalités dans la plupart des zones et le caractère régressif du péage à l'échelle de l'agglomération. L'indicateur de Theil révèle, par contre, une réduction des inégalités et un effet redistributif du péage dans certaines zones et dans l'ensemble du périmètre d'étude. Quant à l'accessibilité aux emplois en voiture pour la population active, la mise en place du péage conduit à une diminution de $13 \%$, avec des différences selon les zones. Alors que dans les zones à l'intérieur du cordon de péage l'accessibilité reste stable ou augmente, dans le reste de l'agglomération, elle diminue, avec des variations selon leur localisation.

Les différents résultats de ce travail de simulation ne convergent pas vers une réponse définitive sur les impacts en matière d'inégalités sociales et spatiales de la mise en place d'un péage pour les automobilistes. Cependant, ils montrent bien les difficultés méthodologiques (choix des indicateurs, mesure du revenu, découpage spatial) à surmonter pour mener des analyses fines et, comme les auteures le suggèrent, le besoin de pouvoir intégrer d'autres éléments dans l'évaluation, tels que le report modal et le financement des infrastructures de transport collectif par le biais du péage urbain.

Le deuxième article porte sur un travail de Cécilia Cruz consacré aux spécificités du transport routier de marchandises «pour compte propre », effectué par des firmes pour la livraison de marchandises (ou, plus 
rarement, de services associés à des productions de haute valeur) produites par elles-mêmes, sans avoir recours à des transporteurs professionnels travaillant «pour compte d'autrui ». Cette activité (le compte propre) se limite à des trafics majoritairement de courte distance. Il y a pourtant des exceptions, dont l'article nous donne quelques illustrations démontrant la singularité de ces pratiques.

Historiquement, le compte propre a reculé par rapport au compte pour autrui dans les prestations de transport, sous l'effet de la tendance générale des entreprises à l'externalisation des fonctions n'entrant pas dans leur core business, une tendance encouragée par une adaptation croissante des transporteurs aux besoins de leurs clients, les « chargeurs » et la réduction concomitante des coûts par le jeu d'économies d'échelle.

À l'évidence, qu'il s'agisse de courte ou (rarement) de plus longue distance, ce sont des logiques tenant à des caractéristiques particulières de production et/ou de distribution qui dictent le choix de la forme de transport, répondant à des impératifs de coût et/ou de qualité de la prestation. Les politiques de marketing (relation au client) des entreprises de production de biens et de services mais aussi la prise en compte des dimensions territoriales de leurs réseaux de distribution expliquent en grande partie le maintien de la livraison en compte propre, particulièrement résistante sur la courte distance. Les illustrations de livraison sur longue distance en compte propre montrent la spécificité et la qualité exigées de la prestation de transport. Elles font cependant figures d'exception, ainsi qu'en attestent les résultats de l'enquête Echo, centrée sur l'analyse méthodique des envois.

Au total, l'article a le mérite de proposer les principaux facteurs d'évolution de ces deux formes juridiquement et économiquement distinctes de la prestation de transport.

Dans cette courte sélection de six articles issus du $48^{\text {e }}$ Colloque de l'ASRDLF, les thématiques autour des liens des mobilités et des systèmes de transport avec les territoires, urbains ou non, sont majoritaires. Mais il est à remarquer que les approches et les interrogations d'ordre méthodologique sont très présentes, permettant ainsi de mener des analyses de plus en plus fines. Si cette année, le $50^{\mathrm{e}}$ Colloque de l'ASRDLF, qui aura lieu à Mons (Belgique), a comme thématique principale Culture, patrimoine, savoirs nous sommes persuadés que les contributions à la session récurrente Accessibilité, mobilité, transport et communication seront toujours nombreuses. 